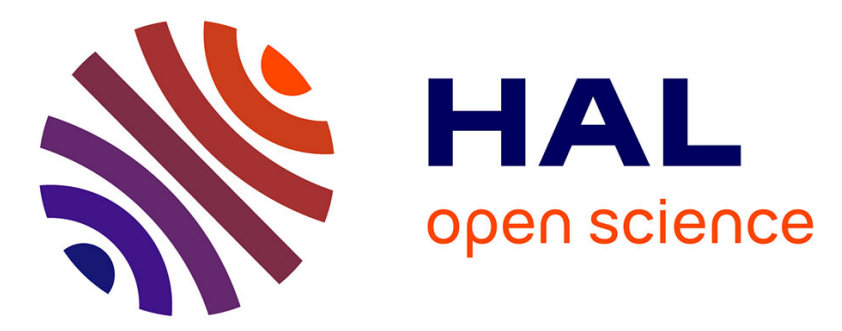

\title{
Assessing non-normal effects in thermoacoustic systems with mean flow
}

Kerstin Wieczorek, Claude Sensiau, Wolfgang Polifke, Franck Nicoud

\section{To cite this version:}

Kerstin Wieczorek, Claude Sensiau, Wolfgang Polifke, Franck Nicoud. Assessing non-normal effects in thermoacoustic systems with mean flow. Physics of Fluids, 2011, 23 (10), pp.107103. 10.1063/1.3650418. hal-00802062

\section{HAL Id: hal-00802062 \\ https://hal.science/hal-00802062}

Submitted on 19 Mar 2013

HAL is a multi-disciplinary open access archive for the deposit and dissemination of scientific research documents, whether they are published or not. The documents may come from teaching and research institutions in France or abroad, or from public or private research centers.
L'archive ouverte pluridisciplinaire HAL, est destinée au dépôt et à la diffusion de documents scientifiques de niveau recherche, publiés ou non, émanant des établissements d'enseignement et de recherche français ou étrangers, des laboratoires publics ou privés. 
Assessing Non-Normal Effects in Thermoacoustic Systems with Mean Flow.

K. Wieczorek, ${ }^{1, \text { a) }}$ C. Sensiau, ${ }^{2}$ W. Polifke, ${ }^{3}$ and F. Nicoud ${ }^{4, b)}$

1) CERFACS CFD-Team, 42 Ave Coriolis, 31057 Toulouse, France

2) SNECMA Villaroche, France

3) Lehrstuhl für Thermodynamik, TU Munich, 85747 Garching, Germany

4) I3M, University Montpellier 2, CC51, 34057 Montpellier Cedex 5, France

( Dated: 20 September 2011) 
In this paper, non-normal interactions in a thermoacoustic system are studied, using a low-order expansion of the state variables in terms of eigenmodes. The thermoacoustic eigenmodes are determined as solutions of the Helmholtz equation or the linearized Euler equations, respectively, in the presence of a time-lagged heat source. Subsequently, non-normal effects are evaluated in a post-processing analysis based on the computed eigenmodes. In the case where the eigenmode analysis is based on the linearized Euler Equations, effects of a non-zero mean flow velocity can be taken into account. The energy associated with the eigenmodes may then contain contributions of convected entropy and vorticity modes as well as the acoustic field. The notion of transient growth of perturbation energy is thus extended from an expression based on the classical acoustic energy density to a form based on a generalized disturbance energy.

The expansion in terms of eigenmodes is computationally efficient, making the approach potentially applicable to complex, 3D configurations including non-trivial boundary conditions and spatio-temporal distributions of heat release fluctuations. In the present paper, the method is applied to a $1 \mathrm{D}$ configuration that consists of a duct including a 1D heat source, followed by a choked isentropic nozzle. It is shown that for such a case it is essential to include the contribution of entropy perturbations in the calculation of the optimal initial perturbation and the maximum transient energy growth. Subsequently, the impact of increasing mean flow Mach number and increasing strength of flame/acoustic interaction on non normal effects is assessed in a parameter study.

a)wieczorek@cerfacs.fr

b)franck.nicoud@univ-montp2.fr 


\section{INTRODUCTION}

Over the last decades, thermoacoustic instabilities have been the subject of intense research activity with the aim to better understand and predict them at the design level, thus avoiding problems in late development or during commissioning. Unless the pertinent governing equations are solved in the time domain (e.g. when Large Eddy Simulation is used), linear stability analysis often relies on a modal approach, where eigenmodes/eigenfrequencies of the thermoacoustic system are sought for. A system is considered linearly stable, if and only if all its modes decay exponentially. However, the eigenmodes are in general not orthogonal, because of boundary conditions and/or coupling between acoustic perturbations and heat release $\mathrm{I}^{1,2}$. Thus the associated eigenfrequencies provide information only about the long-term evolution of the modes. Due to non-normality, linear modes may interact and transient energy growth can be observed even for stable systems. This effect was demonstrated by Balasubramanian \& Sujith ${ }^{2}$ who transferred ideas initially developed for shear flow instabilities ${ }^{3-6}$ to the thermoacoustic context.

The maximum energy growth that can appear depends only on the thermoacoustic system of interest. Calling $\mathcal{U}$ the state vector (typically the components of $\mathcal{U}$ are the fields of acoustic density, pressure and velocity), the relevant equations for describing the time evolution of the perturbations read formally:

$$
\frac{\partial \mathcal{U}}{\partial t}+\mathcal{A}(\mathcal{U})=0
$$

where $\mathcal{A}$ is a differential operator, which is linear when linear thermoacoustics is considered. Eq. (1) is nothing but a set of partial differential equations, which can be reduced to a set of ordinary differential equations when an appropriate discretization method is used. The thermoacoustic system of interest is then represented by a first order dynamical system which reads:

$$
\frac{d \mathbf{u}}{d t}+\mathbf{A u}=0
$$

where $\mathbf{u}$ is the discretized counterpart of $\mathcal{U}$. If Eq. (2) is obtained from Eq. (1) using a modal expansion ("Galerkin") technique, i.e. by expanding the fluctuating quantities in a series of orthogonal basis functions ${ }^{7}$, the vector $\mathbf{u}$ contains the weights of those basis functions. On the other hand, if a finite difference/finite volume technique is used, $\mathbf{u}$ contains the nodal values of the state vector $\mathcal{U}$. Of course, the square matrix A depends on the discretization technique, both in its size and structure. Typically, a modal expansion produces a dense 
matrix of small size, as the expansion requires usually only few basis functions ${ }^{8}$, whereas a finite volume approach produces a large, but sparse matrix ${ }^{9}$.

In any case, some of the characteristics of the thermoacoustic system can be studied by analyzing the matrix $\mathbf{A}$ instead of the differential operator $\mathcal{A}$. Notably, the maximum transient energy growth at time $t$,

$$
G(t)=\max _{\text {initial states }} \frac{E(t)}{E(t=0)},
$$

where $E$ is a suitably defined measure of perturbation energy (see below), is related to the largest singular value of the exponential matrix $\exp (-\mathbf{A} t)^{5}$. This property was used in several recent studies in order to quantify the non-normal effects in simple thermoacoustic systems such as the Rijke tube ${ }^{2}$ or a laminar diffusion flame ${ }^{10}$. This allowed assessing the maximum transient growth $G_{\max }=\max [G(t)]$, the maximum value being taken over all the possible values of $t$. Unfortunately, we believe that this approach based on a Singular Value Decomposition (SVD) of the matrix $\mathbf{A}$ is not very suitable for complex systems for two main reasons:

1. time delay: In practical cases, the flame response to upstream acoustic perturbations is time lagged, the time delay $\tau$ being potentially related to several fluid mechanics and/or chemical processes relevant to the flame unsteadiness. In thermo-acoustic simulations based on linearized equations, this time lag behaviour has to be included explicitly in the system of equations via a model for the heat source. As a consequence, the system cannot be described by Eq. (1), but an expression of the form

$$
\frac{\partial \mathcal{U}}{\partial t}+\mathcal{A}(\mathcal{U}(t))+\mathcal{B}(\mathcal{U}(t-\tau))=0
$$

or generalizations involving several time lags, must be used instead. Unfortunately, generalizing the SVD approach described above to Eq. (4) is not straightforward and may involve additional simplifications like assuming the time delay $\tau$ to be small compared to the first mode's period ${ }^{8}$.

2. boundary conditions: if the Galerkin method is used to convert Eq. (1) into Eq. (2), the knowledge of an orthogonal set of basis functions, which meet the actual boundary conditions of the thermoacoustic problem, is required. Because they convey useful information about the configuration, the acoustic eigenmodes are suitable for this 
purpose $^{7}$. Unfortunately, they are not orthogonal as soon as the boundary conditions correspond to a finite, complex-valued impedance ${ }^{1}$, a situation which is not rare. If a finite difference/finite volume type of approach is used instead, the size of the discretized problem, Eq. (2), is large (typical finite volume grids contain $10^{5}-10^{6}$ elements) so that performing a SVD in order to assess $G(t)$ may be CPU demanding. Thus, maximizing $G(t)$ over all the values of time $t$ to obtain $G_{\max }$ might not be affordable in practice.

Recently, Selimefendigil et al. ${ }^{11}$ proposed a method to handle delayed systems and overcome the first issue mentioned. In their view, Eq. (4) is recast into an equivalent non-delayed problem for which the pseudospectra can be computed. The concept of pseudospectra (first mentioned by Landau ${ }^{12}$ ) was introduced by Trefethen ${ }^{13}$ in order to quantify the sensitivity of eigenvalues to uncertainties/ perturbations in the data or discretization. The property exploited by Selimefendigil et al. ${ }^{11}$ is that the geometry of pseudospectra can be used to obtain a lower bound of $G_{\max }$ (Kreiss theorem). Still, computing pseudospectra becomes very CPU demanding as the size of the problem increases, so that this approach does not address the second issue mentioned. Besides, as far as the authors know, analyzing the pseudospectra can only give information about the maximum transient growth and not about the shape of the optimal perturbation.

The first objective of this paper is to present a strategy, which is potentially suitable for assessing non-normal effects in 3D complex configurations with moderate computational effort. It is based on an expansion in terms of the first few thermoacoustic eigenmodes of the system of interest. Indeed, even if non-normality is present and eigenmodes only provide information about the long term evolution, they convey relevant information about the system. For example, their individual stability dictates the overall stability of the system if non-linear effects are not considered. Numerical strategies have been proposed in the past in order to compute such modes by solving an Helmholtz type of equation with a forcing term representing the flame $e^{1,14,15}$. The view considered in this paper was initially proposed by Schmid \& Henningson ${ }^{5}$ for investigating classical fluid mechanics configurations. It consists in looking for the optimal perturbation (the one which generates the largest transient growth) in the linear space spanned by the thermoacoustics modes. In other words, assessing the non-normality effect amounts to a post-processing of the results of the classical modal characterization of the configuration. As we will demonstrate, this can be 
done at negligible additional cost. Of course, since only a finite number of eigenmodes are considered, all possible initial conditions cannot be generated by combining these modes, thus only a lower bound on $G_{\max }$ can be obtained. However, since the eigenmodes convey a lot of information regarding the system of interest, it is expected that keeping only a few of them is sufficient to obtain a reasonable assessment of the maximum transient growth. The same idea justifies the Galerkin methods where often only a few (of order 10 say) basis functions are necessary to reach good accuracy. However, contrary to the Galerkin method, the orthogonality of the modes is not required in the present approach, so that the method is also suitable for complex 3D configurations with finite, complex-valued boundary impedance.

Practically all of the previous studies dealing with non-normal effects in thermoacoustic systems relied on the zero Mach number mean flow assumption although the effect of the approximation $M \simeq 0$ is not well understood $^{16}$ and the neglected convective terms may introduce additional non-normality ${ }^{17}$. An exception is the study of solid rocket motor instability by Mariappan \& Sujith ${ }^{18}$, where mean flow terms are included in the system of acoustic equations. In the case of a time-lagged heat source (generalized " $n-\tau$ model"), only a moderate non-normal effect has been reported in the literature ${ }^{2}$, with maximum transient energy growth up to $G_{\max } \approx 7$. The second objective of this paper is then to investigate if larger values of $G_{\max }$ can be observed when mean flow effects are not neglected. In this case, the evolution of the perturbations are described by the Linearized Euler Equations (LEE) instead of a simple Helmholtz equation for the acoustic pressure. Also, the state vector contains one more component (the density or entropy, say) on top of the acoustic pressure and velocity fields. Thus this situation is quite different from what has been considered so far and the analysis presented also serves as an illustration of the flexibility of the method and its ability to handle complex situations.

The method is introduced in section II A, giving details on the heat source model and the boundary conditions. Sections II B and IIC then present discussion of a generic 3D thermoacoustic system treated under the zero Mach number assumption. In this case, the state vector contains only the acoustic pressure and velocity fields and non-trivial boundary conditions (finite, complex-valued impedance) can be considered. The formalism is then extended in section IID to the case where the perturbations are obtained from the LEE, and the state vector contains one more component. The method is applied to the simple 
case of a heat source placed in a straight duct. Note however that this situation is more complex than several previous studies since a) a time delayed $n-\tau$ type of model is used for describing the acoustic-flame coupling and b) complex boundary conditions are applied at the boundaries of the duct. The corresponding results are discussed in section III, where the maximum transient growth related to two types of energies is considered, i.e. the classical acoustic energy and the energy of the fluctuations.

\section{FORMALISM}

\section{A. The thermoacoustic model}

The phenomenon of thermoacoustic instability results from a coupling between combustion processes and the acoustic eigenmodes of the configuration ${ }^{19}$. Assuming vanishing Mach number for the mean flow, this coupling can be modeled in the linear regime by the following wave equation :

$$
\frac{1}{\gamma(\mathbf{x}) p_{0}} \frac{\partial^{2} p^{\prime}(\mathbf{x}, t)}{\partial t^{2}}+\nabla \cdot \frac{1}{\rho_{0}(\mathbf{x})} \nabla p^{\prime}(\mathbf{x}, t)=\frac{\gamma(\mathbf{x})-1}{\gamma(\mathbf{x}) p_{0}} \frac{\partial q^{\prime}(\mathbf{x}, t)}{\partial t}
$$

where $p^{\prime}(\mathbf{x}, t)$ stands for the acoustic pressure at position $\mathbf{x}$ and time $t ; \gamma(\mathbf{x})$ and $\rho_{0}(\mathbf{x})$ are the time averaged isentropic coefficient and density of the fluid; $p_{0}$ is the homogeneous background pressure.

Eq. (5) states that heat release fluctuations $q^{\prime}(\mathbf{x}, t)$ may influence the acoustics in the domain. It is common practice to model the feedback effect, viz. the influence of acoustic fluctuations on combustion, via an $n-\tau$ type of model ${ }^{20-22}$. This model assumes that the heat release fluctuations are proportional to the time-lagged velocity fluctuations at a reference point located upstream of the flame:

$$
q^{\prime}(\vec{x}, t)=\frac{q_{t o t}}{u_{b u l k}} H_{q}(\mathbf{x}) \mathbf{u}^{\prime}\left(\mathbf{x}_{r e f}, t-\tau(\mathbf{x})\right) \cdot \mathbf{n}_{r e f},
$$

where $H_{q}(\mathbf{x})$ is the amplitude of the flame response and can be related to the parameter $n$ of $n-\tau$-models ${ }^{9}, \tau(\mathbf{x})$ is the time delay and $\mathbf{n}_{r e f}$ is a unit vector. Assuming timeharmonic perturbations of pulsation $\omega$, one may write $p^{\prime}(\mathbf{x}, t)=\Re\left(\hat{p}(\mathbf{x}) e^{-i \omega t}\right)$ and $q^{\prime}(\vec{x}, t)=$ $\Re\left(\hat{q}(\mathbf{x}) e^{-i \omega t}\right)$. The acoustic field can then be expressed in terms of eigenmodes that are solution of a Helmholtz equation written for the complex amplitude of pressure $\hat{p}$ :

$$
\gamma(\mathbf{x}) p_{0} \nabla \cdot\left(\frac{1}{\rho_{0}(\mathbf{x})} \nabla \hat{p}(\mathbf{x})\right)+\omega^{2} \hat{p}(\mathbf{x})=\frac{q_{t o t}}{i \omega \rho_{r e f} u_{b u l k}} H_{q}(\mathbf{x}) e^{i \omega \tau(\mathbf{x})} \nabla \hat{p}\left(\mathbf{x}_{r e f}\right) \cdot \mathbf{n}_{r e f}
$$


As the problem has been written in frequency domain, the reflection of low frequency waves at the boundaries can be handled easily with a complex-valued impedance at the boundary, denoted $Z(\omega), Z \in \mathbb{C}$. The appropriate boundary condition to impose to $\hat{p}$ takes the following form:

$$
\nabla \hat{p}(\mathbf{x}) \cdot \mathbf{n}_{B C}-i \frac{\omega}{c_{0}(\mathbf{x}) Z(\omega)} \hat{p}(\mathbf{x})=0
$$

where $\mathbf{n}_{B C}$ is a unit vector normal to the boundary and $c_{0}(\mathbf{x})$ is the speed of sound. Solving the eigenproblem given by Eq. (7) and Eq. (8) allows to determine the thermoacoustic

pressure eigenmodes $\hat{p}(\mathbf{x})$, and their corresponding eigenfrequencies $\omega$. The velocity eigenmodes $\hat{\mathbf{u}}(\mathbf{x})$ can then be deduced using the linearised Euler equation written in the frequency domain for time harmonic fluctuations:

$$
i \omega \rho_{0} \hat{\mathbf{u}}(\mathbf{x})=\nabla \hat{p}(\mathbf{x})
$$

\section{B. Non-orthogonality of the eigenfunction}

Non-normality arises from the fact that the thermoacoustic eigenmodes are not orthogonal. Thus, it is important to specify how orthogonality is defined or, equivalently, what is the appropriate inner product. The formalism used throughout this paper is therefore stated in the following.

An acoustic perturbation is defined as a vector composed of pressure and velocity fluctuations $p^{\prime}$ and $\mathbf{u}^{\prime}$ that are assumed to be harmonic in time. This allows to write:

$$
v^{\prime}(\mathbf{x}, t)=\left[\begin{array}{l}
p^{\prime}(\mathbf{x}, t) \\
\mathbf{u}^{\prime}(\mathbf{x}, t)
\end{array}\right]=\left[\begin{array}{l}
\Re\left(\hat{p}(\mathbf{x}) e^{-i \omega t}\right) \\
\Re\left(\hat{\mathbf{u}}(\mathbf{x}) e^{-i \omega t}\right)
\end{array}\right]=\Re\left(\hat{v}(\mathbf{x}) e^{-i \omega t}\right)
$$

where the vector $\hat{v}(\mathbf{x})$ contains the complex amplitudes of pressure and velocity fluctuations, the latter being composed of three components $\hat{\mathbf{u}}(\mathbf{x})=\left(\hat{u}_{x}(\mathbf{x}), \hat{u}_{y}(\mathbf{x}), \hat{u}_{z}(\mathbf{x})\right)$ and $\omega=\omega_{r}+i \omega_{i}$ is a complex frequency.

The following considerations are set in the complex space, i.e. the return to a real-valued vector is dropped. The solutions of the thermoacoustic system are considered in the form 
of complex-valued vectors

$$
v(\mathbf{x}, t)=\hat{v}(\mathbf{x}) e^{-i \omega t}=\hat{v}(\mathbf{x}) e^{-i \omega_{r} t} e^{\omega_{i} t} \quad \text { with } \quad \hat{v}(\mathbf{x})=\left[\begin{array}{c}
\hat{p}(\mathbf{x}) \\
\hat{\mathbf{u}}(\mathbf{x})
\end{array}\right]=\left[\begin{array}{c}
\hat{p}(\mathbf{x}) \\
\hat{u}_{x}(\mathbf{x}) \\
\hat{u}_{y}(\mathbf{x}) \\
\hat{u}_{z}(\mathbf{x})
\end{array}\right]
$$

a complex eigenvector.

Considering $v_{1}(\mathbf{x}, t)$ and $v_{2}(\mathbf{x}, t)$ two complex vectors that are solution of Eqs. (7), (8) and (9), a weighted inner product can be defined as follows:

$$
\left\langle v_{1}(\mathbf{x}, t) \mid v_{2}(\mathbf{x}, t)\right\rangle_{W}=\int_{V}\left(v_{1}(\mathbf{x}, t)^{H} W v_{2}(\mathbf{x}, t)\right) d V
$$

with $v_{1}(\mathbf{x}, t)^{H}=\hat{v}(\mathbf{x})^{H} e^{i \omega_{r} t} e^{\omega_{i} t}$ the conjugate transpose ("Hermitian transpose") of $v_{1}(\mathbf{x}, t)$, $V$ the volume of the domain that is considered and $W$ a weight matrix.

If $W$ is the identity matrix $I$, the inner product defined in Eq. (12) applied to an eigenvector $\hat{v}(\mathbf{x})$ yields simply its $L_{2}$-norm:

$$
\langle\hat{v}(\mathbf{x}) \mid \hat{v}(\mathbf{x})\rangle_{I}=\int_{V} \hat{v}^{H}(\mathbf{x}) I \hat{v}(\mathbf{x}) d V=\|\hat{v}(\mathbf{x})\|_{2}^{2}
$$

By defining the matrix $W$ in an appropriate way, the product of Eq. (12) can be linked to an equivalent of the acoustic energy associated to the mode $v(\mathbf{x})=\hat{v}(\mathbf{x}) e^{-i \omega t}$. For an eigenvector $\hat{v}(\mathbf{x})$ as defined in Eq. (11), a weight matrix

$$
W_{a c}=\left(\begin{array}{cccc}
\frac{1}{2 \gamma p_{0}(\mathbf{x})} & \frac{u_{0 x}}{2 c_{0}^{2}} & \frac{u_{0 y}}{2 c_{0}^{2}} & \frac{u_{0 z}}{2 c_{0}^{2}} \\
\frac{u_{0 x}}{2 c_{0}^{2}} & \frac{\rho_{0}(\mathbf{x})}{2} & 0 & 0 \\
\frac{u_{0 y}}{2 c_{0}^{2}} & 0 & \frac{\rho_{0}(\mathbf{x})}{2} & 0 \\
\frac{u_{0 z}}{2 c_{0}^{2}} & 0 & 0 & \frac{\rho_{0}(\mathbf{x})}{2}
\end{array}\right)
$$

allows to define an equivalent acoustic energy of the form:

$$
\begin{aligned}
E_{a c}(t) & =\langle v(\mathbf{x}, t) \mid v(\mathbf{x}, t)\rangle_{W_{a c}} \\
& =\int_{V}\left(\hat{v}(\mathbf{x})^{H} e^{i \omega_{r} t} e^{\omega_{i} t} W_{a c} \hat{v}(\mathbf{x}) e^{-i \omega_{r} t} e^{\omega_{i} t}\right) d V \\
& =e^{2 \omega_{i} t} \int_{V}\left(\frac{1}{2 \gamma p_{0}(\mathbf{x})}|\hat{p}(\mathbf{x})|^{2}+\frac{\rho_{0}(\mathbf{x})}{2}|\hat{\mathbf{u}}(\mathbf{x})|^{2}+\frac{|\hat{p}(\mathbf{x})|}{c_{0}(\mathbf{x})^{2}}\left|\mathbf{u}_{\mathbf{0}}(\mathbf{x}) \cdot \hat{\mathbf{u}}(\mathbf{x})\right|\right) d V
\end{aligned}
$$

where $|\ldots|$ denotes the absolute value of a complex number. The term $E_{a c}(t)$ is a realvalued energy that is defined based on complex quantities. It shares the same coefficients 
as the classical acoustic energy. Still, it differs from the latter in the sense that the classical acoustic energy is based on the real parts of the complex signals and will hence be noted $E_{a c, \Re}(t)$ in the following:

$$
E_{a c, \Re}(t)=\int_{V}\left(\frac{1}{2 \gamma p_{0}(\mathbf{x})} p^{\prime}(\mathbf{x}, t)^{2}+\frac{\rho_{0}(\mathbf{x})}{2} \mathbf{u}^{\prime}(\mathbf{x}, t)^{2}+\frac{p^{\prime}(\mathbf{x}, t)}{c_{0}(\mathbf{x}, t)^{2}} \mathbf{u}_{0}(\mathbf{x}) \cdot \mathbf{u}^{\prime}(\mathbf{x}, t)\right) d V
$$

Please note that the energy terms of Eq. (14) and (15) do not follow the same temporal evolution. The term of Eq. (14) is defined in a way as to describe the energy of a linear combination of several modes with possibly incommensurate frequencies, which would prohibit time averaging over one period of oscillation.

This difference becomes clearer if one considers the two energy terms defined for orthogonal modes, i.e. of the form $E_{a c}(t)=\left\langle\sum_{j=1}^{m} v_{j}(\mathbf{x}, t) \mid \sum_{j=1}^{m} v_{j}(\mathbf{x}, t)\right\rangle_{W_{a c}}$. For a characteristic time scale of amplification $\theta$ that is much larger than the period of the eigenmodes $T_{j}$ (i.e. for modes with an imaginary frequency much smaller than the real frequency $\omega_{i}<<\omega_{r}$ ), one may link the energy terms in the following way:

$$
E_{a c}(\theta)=\sum_{j=1}^{m} \frac{1}{T_{j}} \int_{0}^{T_{j}} E_{a c, \Re}(\theta, t) d t
$$

In particular, one may consider the case of a domain with a reactive impedance boundary condition, where the eigenmodes are marginally stable. This boundary condition allows an instantaneous flux of acoustic energy across the border of the domain, which means that $E_{a c, \Re}(t)$ will vary. The time-integrated flux over one period of oscillation is zero, though, and the variation in $E_{a c, \Re}(t)$ is not to be attributed to non-orthogonality. As shown by Eq. (16), the equivalent acoustic energy $E_{a c}(t)$ does not reflect that effect and remains constant. Complete equivalence between the terms of Eq. (14) and (15) may thus be established by time averaging. However, in the present case the short term transient behaviour is to be evaluated. Therefore, it does not make sense to introduce a time average.

The orthogonality of the eigenmodes can now be discussed using the inner product introduced in Eq. (12) together with the weight matrix of Eq. (13). The projection of $\hat{v}_{1}(\mathbf{x})$ onto $\hat{v}_{2}(\mathbf{x})$ can be expressed analytically and leads (after some algebra) to the following equation (see Appendix A for a short derivation):

$$
\left\langle\hat{v}_{1} \mid \hat{v}_{2}\right\rangle_{W_{a c}}=\frac{1}{2} \frac{1}{\omega_{1}-\omega_{2}^{*}}\left[\int_{S} \frac{1}{\rho_{0}}\left(\hat{p}_{1} \frac{\nabla \hat{p}_{2}^{*}}{\omega_{2}^{*}}-\hat{p}_{2}^{*} \frac{\nabla \hat{p}_{1}}{\omega_{1}}\right) \cdot \mathbf{n} d S+i \int_{V} \frac{\gamma-1}{\gamma p_{0}}\left(\hat{p}_{1} \hat{q}_{2}^{*}+\hat{p}_{2}^{*} \hat{q}_{1}\right) d V\right]
$$


where $S$ and $V$ denote the surface and the volume of the domain, respectively, and * stands for complex conjugates. (The dependency of the complex variables on $\mathbf{x}$ is omitted for clarity, and the mean flow speed is neglected.)

The expression of Eq. (17) is an extension of the result by Nicoud et al. ${ }^{1}$, who were considering only pressure fluctuations in their analysis of the eigenmodes' orthogonality, instead of the complete mode structure composed of pressure and velocity terms. Eq. (17) shows that two eigenmodes $\hat{v}_{1}$ and $\hat{v}_{2}$ are orthogonal when a) boundary impedances are trivial , i.e. they correspond to pressure or velocity nodes with $\hat{p}=0$ or $\nabla \hat{p}=0$ respectively; and b) no heat release fluctuations occur $(\hat{q}=0)$. However the conditions for orthogonality will most probably never be met in an actual experimental setup, so that non-normality should be considered as the rule for practical thermoacoustic systems.

As a consequence, even if all the eigenmodes are found stable $\left(\omega_{i}<0\right)$, there is a possibility for the equivalent acoustic energy of Eq. (14) to exhibit transient growth before it eventually vanishes as predicted by linear modal analysis. The amplitude of the acoustic fluctuations may become significant during this transient phase and the linear assumption is possibly not valid anymore. In particular, it has been shown ${ }^{23}$ that gain and phase of the flame transfer function may depend significantly on the amplitude of the velocity fluctuations. This is the reason why non-normality is sometimes related to complex effects such as non-linear triggering ${ }^{2}$.

The focus of the present study is however limited to the assessment of non-normality effects in complex configurations and non-linearity is not considered.

\section{The maximum possible amplification}

For complex time-dependant signals of pressure and velocity $q(\mathbf{x}, t)$, a maximum possible amplification $G_{a c}(t)$ can be defined as

$$
G_{a c}(t)=\max \frac{E_{a c}(t)}{E_{a c}(0)}=\max _{q(\mathbf{x}, 0) \neq 0} \frac{\langle q(\mathbf{x}, t) \mid q(\mathbf{x}, t)\rangle_{W_{a c}}}{\langle q(\mathbf{x}, 0) \mid q(\mathbf{x}, 0)\rangle_{W_{a c}}}
$$

This quantity should be thought of as the upper bound of the envelop of the equivalent acoustic energy. Starting from any perturbation with a unit energy norm, the equivalent acoustic energy term will always remain smaller than or equal to this coefficient: $E_{a c}(t)<$ 
$G_{a c}(t), \forall t$. Still, there is no reason why the optimal perturbation, which maximizes $E_{a c}$ at time $t_{1}$, should also maximize $E_{a c}$ at time $t_{2} \neq t_{1}$, thus the envelop.

Schmidt \& Henningson ${ }^{5}$ provide a procedure to assess this maximum possible amplification for complex signals $q(\mathbf{x}, t)$ that can be expressed as a linear combination of $m$ complex eigenmodes:

$$
q(\mathbf{x}, t)=\sum_{j=1}^{m} k_{j} \hat{v}_{j}(\mathbf{x}) e^{-i \omega_{j} t}
$$

This expansion of $q(\mathbf{x}, t)$ as a linear combination of eigenmodes can be rewritten in a compact matrix notation as

$$
q(\mathbf{x}, t)=\hat{V}(\mathbf{x}) e^{-i \Omega t} \mathbf{k}
$$

where the $j^{\text {th }}$ column of the matrix $\hat{V}(\mathbf{x})$ contains the complex-valued eigenvector $\hat{v}_{j}(\mathbf{x})$, the diagonal matrix $\Omega=\operatorname{diag}\left(\omega_{1}, \omega_{2}, \ldots, \omega_{m}\right)$ contains the complex frequencies of the $m$ eigenvectors used for the expansion and the vector $\mathbf{k}$ stores the coefficients $k_{j}$ of the linear combination Eq. (19).

Introducing Eq. (20) into the definition of the equivalent acoustic energy of Eq. (14), one obtains:

$$
\begin{aligned}
E_{a c}^{m}(t) & =\langle q(\mathbf{x}, t) \mid q(\mathbf{x}, t)\rangle_{W_{a c}} \\
& =\int_{V} q(\mathbf{x}, t)^{H} W_{a c} q(\mathbf{x}, t) d V \\
& =\int_{V}\left(e^{-i \Omega t} \mathbf{k}\right)^{H} \hat{V}(\mathbf{x})^{H} W_{a c} \hat{V}(\mathbf{x}) e^{-i \Omega t} \mathbf{k} d V \\
& =\left(e^{-i \Omega t} \mathbf{k}\right)^{H} M_{a c}\left(e^{-i \Omega t} \mathbf{k}\right)
\end{aligned}
$$

where the matrix $M$ contains the inner products of the $m$ selected eigenvectors:

$$
M_{a c}=\int_{V}\left(\hat{V}(\mathbf{x})^{H} W_{a c} \hat{V}(\mathbf{x})\right) d V=\langle\hat{V}(\mathbf{x}) \mid \hat{V}(\mathbf{x})\rangle_{W_{a c}}
$$

The element $k l$ of this matrix reads:

$$
M_{a c}^{k l}=\int_{V}\left(\hat{v}_{k}(\mathbf{x})^{H} W_{a c} \hat{v}_{l}(\mathbf{x})\right) d V
$$

Since $\langle\cdot \mid \cdot\rangle_{W_{a c}}$ is an inner product, $M_{a c}$ is a positive Hermitian matrix so that its Cholesky decomposition exists and yields the square matrix $F_{a c}$ of size $m$ such that $F_{a c}^{H} F_{a c}=M_{a c}$. Introducing the decomposition of $M_{a c}$ into the acoustic energy term of Eq. (21) one obtains:

$$
E_{a c}^{m}(t)=\left(F_{a c} e^{-i \Omega t} \mathbf{k}\right)^{H}\left(F_{a c} e^{-i \Omega t} \mathbf{k}\right)
$$


This equation shows that $E_{a c}(t)$ is nothing but the $L_{2}$-norm of the vector $F_{a c} e^{-i \Omega t} \mathbf{k}$. Note that in difference to the energy term defined for one single mode (Eq. (14)), the equivalent acoustic energy term for a superposition of several modes is function not only of $\omega_{i}$, but also of $\omega_{r}$ (via the matrix $\Omega$ ).

Finally, noting that the Cholesky factor $F_{a c}$ is not singular, the maximum possible amplification at time $t$ takes the following form:

$$
G_{a c}^{m}(t)=\max \frac{E_{a c}^{m}(t)}{E_{a c}^{m}(0)}=\max \frac{\left\|F_{a c} e^{-i \Omega t} \mathbf{k}\right\|_{2}^{2}}{\left\|F_{a c} \mathbf{k}\right\|_{2}^{2}}=\max _{F_{a c} \mathbf{k}} \frac{\left\|F_{a c} e^{-i \Omega t} F_{a c}^{-1} F_{a c} \mathbf{k}\right\|_{2}^{2}}{\left\|F_{a c} \mathbf{k}\right\|_{2}^{2}}
$$

By definition, this quantity is the $L_{2}$-norm of the operator $F_{a c} e^{-i \Omega t} F_{a c}^{-1}$. In other words, the maximum amplification at time $t$ is given by the largest singular value of $F_{a c} e^{-i \Omega t} F_{a c}^{-1}$. The optimal initial perturbation is given by the corresponding right singular vector of $F_{a c} e^{-i \Omega t} F_{a c}^{-1}$.

On the LHS of Eq. (25), the superscript $m$ indicates that this expression gives the maximum energy amplification at time $t$ for all the perturbations which can be obtained by combining the $m$ selected eigenvectors (this notation is sufficient if one assumes that the $m$ vectors selected correspond to the $m$ lowest eigenfrequencies). In the same way, the maximum transient growth, which can be obtained by combining these $m$ eigenvectors, can be obtained by maximizing $G_{a c}^{m}(t)$ over time and shall be noted:

$$
G_{\max , a c}^{m}=\max _{t} G_{a c}^{m}(t)
$$

In the case where the eigenmodes are orthogonal and all damped, the matrices $M_{a c}$ and $F_{a c}$ are both diagonal. Then, Eq. (25) shows that the maximum growth rate equals unity (because $F_{a c} e^{-i \Omega t} F_{a c}^{-1}$ reduces to $e^{-i \Omega t}$ ) as it is expected when non-normality is not present. As a last comment, we stress the fact that the singular value decomposition is performed on a matrix of size $m$ (which is the number of eigenmodes used to generate the signal), making the above approach computationally inexpensive.

\section{Extension to non isentropic modes}

When the LEE equations are solved instead of the Helmholtz equation for pressure only, the thermoacoustic modes contain one more component, in the presented case the fluctuating 
entropy. Any mode can thus be represented with the following compact notation :

$$
v(\mathbf{x}, t)=\hat{v}(\mathbf{x}) e^{-i \omega t}=\hat{v}(\mathbf{x}) e^{-i \omega_{r} t} e^{\omega_{i} t} \quad \text { with } \quad \hat{v}(\mathbf{x})=\left[\begin{array}{c}
\hat{p}(\mathbf{x}) \\
\hat{\mathbf{u}}(\mathbf{x}) \\
\hat{s}(\mathbf{x})
\end{array}\right]=\left[\begin{array}{c}
\hat{p}(\mathbf{x}) \\
\hat{u}_{x}(\mathbf{x}) \\
\hat{u}_{y}(\mathbf{x}) \\
\hat{u}_{z}(\mathbf{x}) \\
\hat{s}(\mathbf{x})
\end{array}\right]
$$

To describe the energy contained in this kind of modes, the corollary for disturbance energy derived by Myers $^{24}$ and extended by Karimi et al. ${ }^{25}$ is appropriate. Thus, instead of the classical acoustic energy of Eq. (15), the following term should be used to determine the total energy of the disturbances:

$E_{t o t, \Re}=\int_{V}\left(\frac{1}{2 \gamma(\mathbf{x}) p_{0}} p^{\prime}(\mathbf{x}, t)^{2}+\frac{\rho_{0}(\mathbf{x})}{2} \mathbf{u}^{\prime}(\mathbf{x}, t)^{2}+\frac{\rho_{0}(\mathbf{x}) T_{0}(\mathbf{x})}{2 C_{p}(\mathbf{x})} s^{\prime}(\mathbf{x}, t)^{2}+\rho^{\prime}(\mathbf{x}, t) \mathbf{u}_{0}(\mathbf{x}) \cdot \mathbf{u}^{\prime}(\mathbf{x}, t)\right) d V$

The weight matrix $W_{\text {tot }}$ that relates the inner product of Eq. (12) to a complex based equivalent of the total disturbance energy of Eq. (28) reads

$$
W_{t o t}=\left(\begin{array}{ccccc}
\frac{1}{2 \gamma p_{0}} & \frac{u_{0 x}}{2 c_{0}^{2}} & \frac{u_{0 y}}{2 c_{0}^{2}} & \frac{u_{0 z}}{2 c_{0}^{2}} & 0 \\
\frac{u_{0 x}}{2 c_{0}^{2}} & \frac{\rho_{0}}{2} & 0 & 0 & -\frac{\rho_{0} u_{0 x}}{2 C_{p}} \\
\frac{u_{0 y}}{2 c_{0}^{2}} & 0 & \frac{\rho_{0}}{2} & 0 & -\frac{\rho_{0} u_{0 y}}{2 C_{p}} \\
\frac{u_{0 z}}{2 c_{0}^{2}} & 0 & 0 & \frac{\rho_{0}}{2} & -\frac{\rho_{0} u_{0 z}}{2 C_{p}} \\
0 & -\frac{\rho_{0} u_{0 x}}{2 C_{p}} & -\frac{\rho_{0} u_{0 y}}{2 C_{p}} & -\frac{\rho_{0} u_{0 z}}{2 C_{p}} & \frac{\rho_{0} T_{0}}{2 C_{p}}
\end{array}\right)
$$

The resulting energy term reads then

$$
\begin{aligned}
E_{t o t}(t) & =\langle v(\mathbf{x}, t) \mid v(\mathbf{x}, t)\rangle_{W_{t o t}} \\
& =e^{2 \omega_{i} t} \int_{V}\left(\frac{1}{2 \gamma p_{0}}|\hat{p}|^{2}+\frac{\rho_{0}}{2}|\hat{\mathbf{u}}|^{2}+\frac{\rho_{0} T_{0}}{2 C_{p}}|\hat{s}|^{2}+\left(\frac{\hat{p}}{c_{0}^{2}}-\frac{\rho_{0}}{C_{p}} \hat{s}\right)\left|\mathbf{u}_{\mathbf{0}} \cdot \hat{\mathbf{u}}\right|\right) d V
\end{aligned}
$$

where the dependencies on $\mathbf{x}$ were omitted for clarity.

The energy term based on complex quantities (Eq. (30)) is formally equivalent to the one based on real-valued quantities (Eq. (28)), as the last term of the RHS integral can be rewritten using the linearized state equation

$$
\hat{\rho}=\frac{\hat{p}}{c_{0}^{2}}-\frac{\rho_{0}}{C_{p}} \hat{s}
$$


It can be shown ${ }^{26}$ that the matrix $W_{\text {tot }}$ defined in Eq. (29) is definite positive as long as the local mean flow Mach number is smaller than a critical value, more precisely if $M a=\sqrt{\mathbf{u}_{\mathbf{0}} \cdot \mathbf{u}_{\mathbf{0}}} / c_{0}<1 / \gamma$. Since this condition is well satisfied for practical combustion systems, the following integral:

$$
\left\langle\hat{v}_{1}(\mathbf{x}) \mid \hat{v}_{2}(\mathbf{x})\right\rangle_{W_{\text {tot }}}=\int_{V}\left(\hat{v}_{1}(\mathbf{x})^{H} W_{\text {tot }} \hat{v}_{2}(\mathbf{x})\right) d V
$$

defines an inner product. Thus, the analytical development described in section II C remains valid in the non-isentropic case and the maximum growth at time $t$ can be written as:

$$
G_{t o t}^{m}(t)=\max \frac{E_{t o t}^{m}(t)}{E_{\text {tot }}^{m}(0)}=\max _{F_{\text {tot }} \mathbf{k}} \frac{\left\|F_{\text {tot }} e^{-i \Omega t} F_{\text {tot }}^{-1} F_{t o t} \mathbf{k}\right\|_{2}^{2}}{\left\|F_{\text {tot }} \mathbf{k}\right\|_{2}^{2}}=\left\|F_{\text {tot }} e^{-i \Omega t} F_{\text {tot }}^{-1}\right\|_{2}^{2}
$$

where the Cholesky factorisation of the matrix

$$
M_{\text {tot }}=\int_{V}\left(\hat{V}(\mathbf{x})^{H} W_{t o t} \hat{V}(\mathbf{x})\right) d V=F_{t o t}^{H} F_{t o t}
$$

has been introduced. This decomposition exists, if $W_{\text {tot }}$ is symmetric positive definite ${ }^{26}$, i.e. when $M a<1 / \gamma$. It might also exist even if the latter condition is not met locally, in a choked nozzle say, where the local mean Mach number is obviously greater than the critical value. This is due to the volume integral in the definition of $M_{\text {tot }}$, which allows some compensation between low and large Mach number regions. Similarly to the isentropic case of section II C, the maximum energy at time $t$ is given by the largest singular value of $F_{t o t} e^{-i \Omega t} F_{t o t}^{-1}$ and the corresponding initial perturbation is given by the right principal singular vector. The maximum transient growth which can be obtained by combining $m$ eigenvectors is:

$$
G_{m a x, t o t}^{m}=\max _{t} G_{t o t}^{m}(t) .
$$

As in the isentropic case of section II C, we may remark that :

- Eq. (32) produces $G_{m a x, t o t}^{m}=1$ when the eigenmodes are orthogonal and damped,

- the requested SVD is still to be made on a matrix of size $m$, thus not very CPUdemanding

The theoretical results established in sections II C and II D are now used to study an academic configuration where Mach number effects are present. 


\section{RESULTS}

\section{A. Configuration}

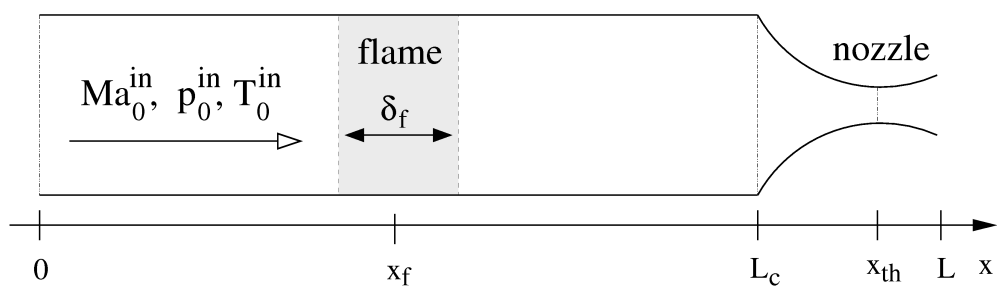

FIG. 1. The numerical setup to assess non-normality with non-zero mean flow (from Nicoud and Wieczorek $\left.^{9}\right)$.

\begin{tabular}{|ccccccc|}
\hline$L(\mathrm{~m})$ & $L_{c}(\mathrm{~m})$ & $x_{\text {throat }}(\mathrm{m})$ & $x_{f}(\mathrm{~m})$ & $\delta_{f}(\mathrm{~m})$ & $\gamma$ & $r(\mathrm{~S} . \mathrm{I})$ \\
1.1 & 1.0 & 1.0863 & 0.5 & 0.15 & 1.4 & 287 \\
\hline \hline$p_{0}^{\text {in }}(\mathrm{Pa})$ & $T_{0}^{\text {in }}=T_{u}(\mathrm{~K})$ & $T_{b}(\mathrm{~K})$ & $M_{0}^{\text {in }}$ & $M_{0}^{\text {out }}$ & $x_{\text {ref }}(\mathrm{m})$ & \\
101325 & 300 & 1200 & 0.05 & 1.5 & 0.42 & \\
\hline
\end{tabular}

TABLE I. Main physical parameters used for configuration of Fig. 1.

The numerical setup consists in a duct of constant cross section of length $L_{c}$, with a region of heat release ("flame") of axial extent $\delta_{f}$ located at $x=x_{f}$ and connected to a nozzle of length $L-L_{c}$ (see Fig. 1). As in ${ }^{9}$, the mean flow is assumed isentropic except in the region of heat release and is constructed from analytical expressions of the temperature profile in the combustion chamber and the Mach number distribution in the isentropic nozzle as follows:

$$
\begin{aligned}
& T_{0}(x)=\frac{T_{0}^{\text {out }}+T_{0}^{\text {in }}}{2}+\frac{T_{0}^{\text {out }}-T_{0}^{\text {in }}}{2} \tanh \left(3 \frac{x-x_{f}}{\delta_{f} / 2}\right) \\
& M a_{0}(x)=M a_{0}^{\text {in }}+\left(M a_{0}^{\text {out }}-M a_{0}^{\text {in }}\right)\left(\frac{x-L_{c}}{L-L_{c}}\right)^{3}
\end{aligned}
$$

The mean flow is then entirely determined by the choice of three independent inlet quantities (for example $p_{0}^{\text {in }}, T_{0}^{\text {in }}, M_{0}^{\text {in }}$ ), the outlet Mach number $M_{0}^{\text {out }}$ and relevant geometrical parameters $\delta_{f}, x_{f}, L_{c}, x_{\text {throat }}$ and $L$. 
The mean profiles depicted in Fig. 2 correspond to the numerical values gathered in Table I and used throughout the course of this study. $T_{u}$ and $T_{b}$ are the temperature of cold ("unburnt") and hot ("burnt") gas on both sides of the heat source, respectively. Note that in the presented case, the gain of the transfer function $H_{q}$ of Eq. (6) is set to zero, i.e. unsteady heat release is not considered. However, there is still interaction of the acoustic field with the heat source, as acoustic perturbations generate entropy waves in the zone of non-zero heat release, which are then convected downstream and may in turn create acoustic waves at the nozzle, which then propagate back into the duct ${ }^{9,27}$.

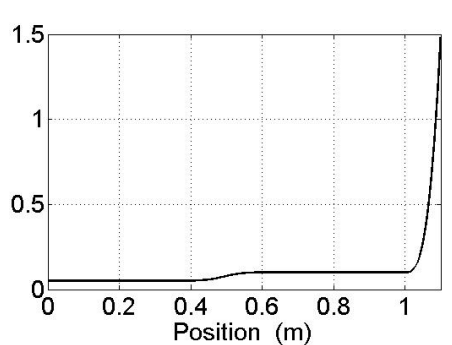

(a)Mach number

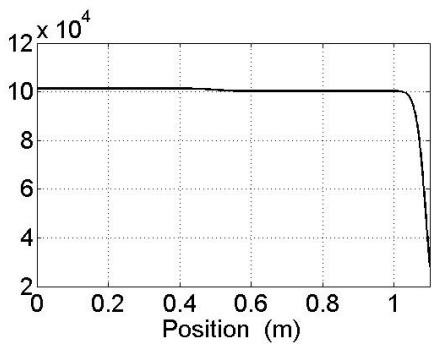

(b)Static pressure $(\mathrm{Pa})$

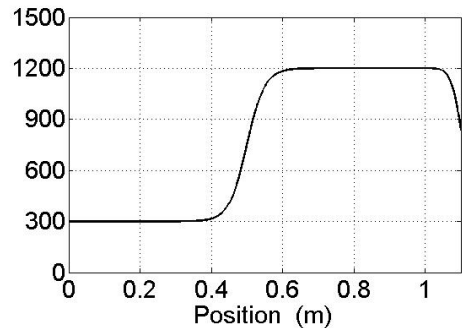

(c) Static temperature $(\mathrm{K})$

FIG. 2. Mean flow fields for the configuration of Fig. 1.

The first eigenmodes of the configuration are computed following the procedure described in $^{9}$ where the Linearized Euler Equations written in the frequency space are discretized on a staggered mesh. The first three modes are displayed in Fig. 3 where the modulus of the complex amplitudes of pressure, velocity and entropy are plotted.

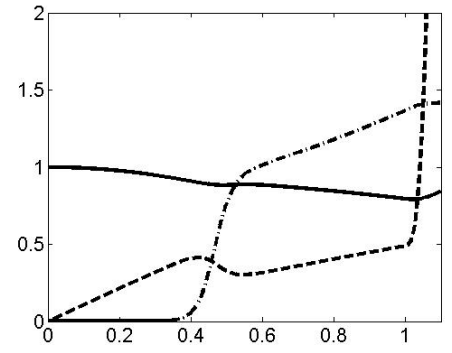

(a)Mode 1 at $59.1-8.3 i \mathrm{~Hz}$

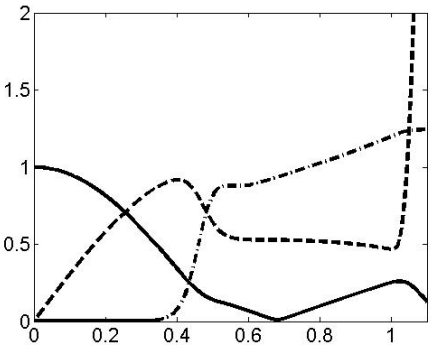

(b)Mode 2 at $169.6-9.4 i \mathrm{~Hz}$

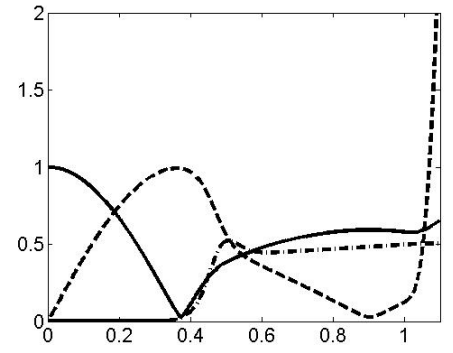

(c) Mode 3 at $231.7-4.3 i \mathrm{~Hz}$

FIG. 3. The first 3 modes in the configuration of Fig. 1. - $:|\hat{p}(x)| ; \quad---:|\hat{u}(x)| ; \quad-\cdot-$ : $|\hat{s}(x)|$. The fluctuating quantities are scaled by $\gamma p_{0}, c_{0}$ and $10 \times C_{v}$ respectively. 


\section{B. Transient Energy Growth \& Optimal Initial Perturbation}

Based on the first six eigenmodes obtained from solving the Linearized Euler Equations, the optimal initial perturbation and the corresponding transient growth are determined. As explained in section II, two alternative definitions of "perturbation energy" are used, based on the classical acoustic energy $G_{a c}^{m}(t)$ as noted in Eq. (25), and on the total disturbance energy $G_{t o t}^{m}(t)$ as in Eq. (32), respectively. As the configuration allows for the presence of entropy fluctuations, the optimal initial perturbation may include acoustic and entropy fluctuations in both cases (see Fig. 5). The important difference between $G_{a c}^{m}(t)$ and $G_{t o t}^{m}(t)$ consists in the fact that the contribution of entropy fluctuations to the energy term are considered negligible in the former approach, whereas they are taken into account in the latter.

It should be noted that the evaluation based on $E_{a c}(t)$ alongside with $E_{t o t}(t)$ is included here in order to point out the importance of the correct choice of the energy form. The evaluation of non-orthogonal effects as post-processing of an eigenvalue analysis may be more prone to inconsistencies than other approaches. The following demonstration is therefore meant to caution the reader against misleading results, which occur if the presented technique is used inadequately.

The temporal evolution of the terms $G_{a c}^{6}(t)$ and $G_{t o t}^{6}(t)$ is shown in Fig. 4(a). In this plot, the time is scaled by the period of the first eigenmode, which has a frequency of $f_{1}=59.1-8.3 i \mathrm{~Hz}$ (cf. Fig. 3(a)); the possible transient energy growth is plotted using a $\log$-scale. It is obvious that the two quantities behave very differently, their maximal values being $G_{\text {max }, a c}^{6} \approx 6000$ and $G_{\text {max,tot }}^{6} \approx 6$. In both cases, however, the maximum possible

amplification is reached at a reduced time of $t_{\max }^{\prime} \approx 0.5$, i.e. after half a period of the first mode. It should also be noted that at very low Mach numbers the two approaches lead to results comparable with each other, with values of $G_{\text {max }, t o t}^{m} \approx G_{m a x, a c}^{m} \approx 1$ (not shown).

Fig. 4(b) shows the temporal evolution of $G_{\text {tot }}^{6}(t)$ together with that of the energy of the optimal initial perturbation $E_{o p t, t o t}^{6}(t)$. The energy of the initial perturbation follows very closely the curve of $G(t)$. This indicates that the optimal initial condition maximises the energy of the system not only at $t=t_{\max }$, but rather throughout the complete initial phase considered here. 


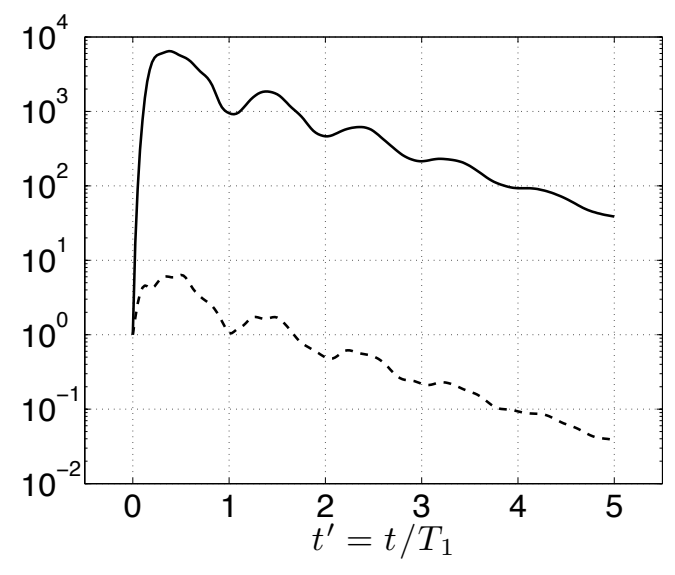

$(\mathrm{a})-G_{a c}^{6}(t) ; \quad---G_{t o t}^{6}(t)$

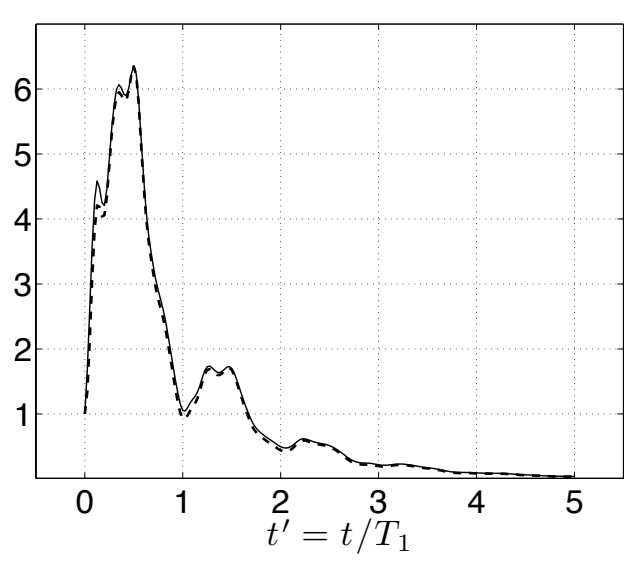

(b) $-G_{t o t}^{6}(t) ; \quad---E_{o p t, t o t}^{6}(t)$

FIG. 4. Temporal evolution of the maximum possible amplification and energy term as obtained from the first six eigenmodes. Time is scaled by the period of the first eigenmode $t^{\prime}=\frac{t}{T_{1}}$ with $f_{1}=59.1-8.3 i \mathrm{~Hz}$.

The optimal initial perturbations that allow to obtain the maximum possible amplifications $G_{a c}^{6}(t)$ and $G_{\text {tot }}^{6}(t)$ are shown in Fig. 5 for $t=0$. Figure 6 shows the same perturbations at the moment of maximum possible amplification, i.e. at $t=t_{\max }$.

In the optimal initial perturbation obtained using the total energy approach (see Fig. 5(b)), fluctuations of entropy, pressure and velocity are equally present. At the time of maximum growth $t=t_{\max }$, the entropy contribution to the disturbance energy term $E_{\text {tot }}$ has increased significantly, while the acoustic mode persists (see Fig. 6(b)). The situation is rather different for the optimal perturbation computed based on the acoustic energy only. At the initial time, the optimal perturbation contains mainly entropy fluctuations, the acoustic contribution being virtually zero (see Fig. $5(\mathrm{a})$ ). However, at $t=t_{\max }$ the entropy fluctuations have decreased, while the acoustic part has increased significantly (see Fig. 6(a)). This means that energy has been transferred from entropy towards acoustic fluctuations. However, as entropy fluctuations were not taken into account in the computation of the energy term, this also means that the acoustic energy term $E_{a c}$ is amplified enormously as it grows from an initial value close to zero to a non-zero value at $t=t_{\max }$.

This observation is consistent with the fact that a large value of $G_{\text {max }, a c}^{6}$ is observed in Fig. 4, while the value of $G_{\max , t o t}^{6}$ is a lot smaller. 


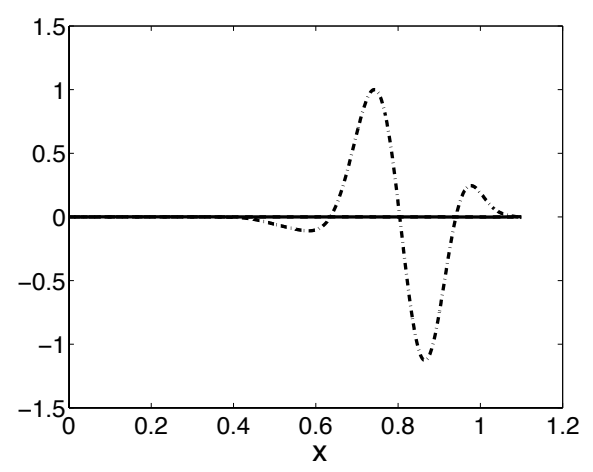

(a)optimal perturbation for $G_{\mathrm{ac}}^{6}(0)$

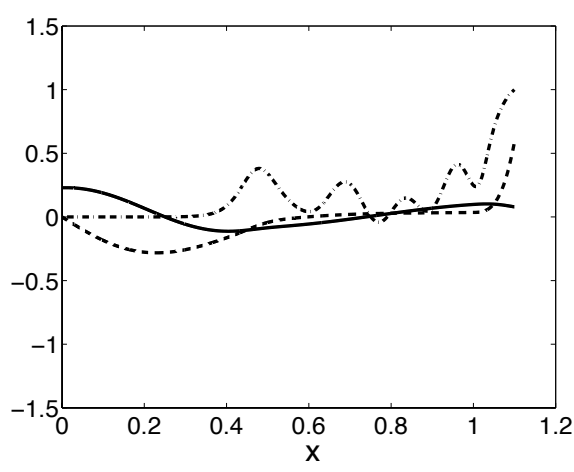

(b)optimal perturbation for $G_{\text {tot }}^{6}(0)$

FIG. 5. Spatial distribution of the optimal initial perturbation at $t=0:-p^{\prime}(x, t) ; \quad---u^{\prime}(x, t)$; - - $s^{\prime}(x, t)$. The fluctuating quantities are scaled by $\gamma p_{0}, c_{0}$ and $C_{v}$ respectively.

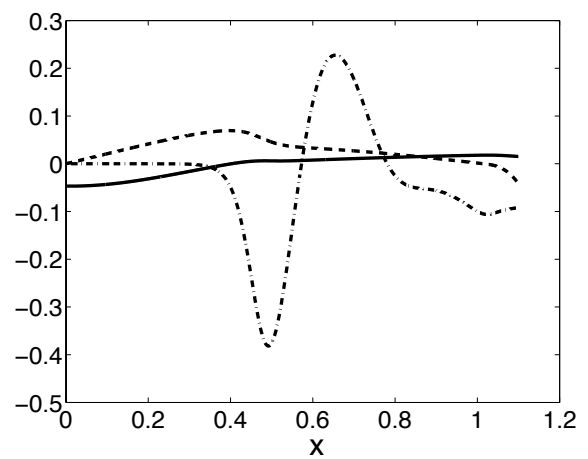

(a)optimal perturbation for $G_{\mathrm{ac}}^{6}\left(t_{\max }\right)$

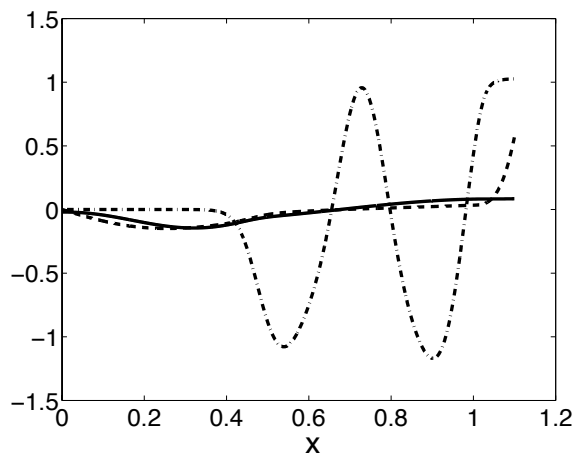

(b)optimal perturbation for $G_{\text {tot }}^{6}\left(t_{\max }\right)$

FIG. 6. Spatial distribution of the optimal perturbation at $t=t_{\max } .-p^{\prime}(x, t) ; \quad---u^{\prime}(x, t)$; - - $s^{\prime}(x, t)$. The fluctuating quantities are scaled by $\gamma p_{0}, c_{0}$ and $C_{v}$ respectively. Note the different scale of the ordinates.

To better understand the physical background of the difference between $G_{\text {max }, a c}^{m}$ and $G_{\max , t o t}^{m}$, the convergence of these quantities with respect to the number of eigenmodes $m$ used for the analysis is displayed in Fig. 7. From Fig. 7(a) one may conclude that the maximum transient amplification is well predicted with only 5-6 modes, when the total energy of the disturbances is considered. Adding more modes to the analysis does not have a huge impact on the result for $G_{m a x, t o t}^{m}$. By contrast, for the growth rate based on the acoustic energy $G_{\max , a c}^{m}$ convergence is hardly reached when 10 modes are used. It seems that the values of $G_{\max , a c}^{m}$ would increase even more when a larger number of eigenmodes is retained. 
This observation is confirmed by Fig. 7(b), which shows the contribution of acoustic fluctuations to the optimal initial perturbation computed with the norm based on the acoustic energy (cf. Figs. 5(a) and 6(a)). For this plot, the value $\sigma(t)$ has been defined as the ratio of acoustic energy to total disturbance energy:

$$
\sigma(t)=\frac{E_{a c}(t)}{E_{t o t}(t)},
$$

where $E_{a c}(t)$ and $E_{t o t}(t)$ are the terms defined in Eq. (14) and (30) respectively. Values of $\sigma(t)$ close to one indicate hence preponderance of acoustic fluctuations and negligible influence of entropy fluctuations, while values of $\sigma(t)$ near zero denote huge contributions of entropy

fluctuations in the signal. For the optimal perturbation corresponding to $G_{\text {max }, a c}^{m}$ at $t=0$, the contribution of the acoustic energy to the total energy clearly tends to zero for increasing $m$ (o-symbols). At the same time, the contribution of acoustic energy to the perturbation at $t=t_{\max }$ remains of the same order of magnitude ( $\times$-symbols). The acoustic transient growth $G_{a c}^{m}(t)=\max E_{a c}(t) / E_{a c}(0)$ is hence virtually unlimited for increasing values of $m$, as $E_{a c}\left(t_{\text {max }}\right)$ does not decrease in the same way as $E_{a c}(0)$.

This behaviour is possible, since the entropy mode of fluctuations can feed the acoustic mode when the mean flow is accelerated ${ }^{9,27,28}$. Another path from entropy to acoustic was discussed by Nicoud and Poinsot ${ }^{29}$ in the case where the thermal diffusivity is not zero. The very large value of $G_{m a x, a c}^{6}$ displayed in Fig. 4 is merely the consequence of these physical phenomena. In other words, non-normality effects cannot be characterized by the transient growth of acoustic energy when either mean flow or thermal diffusivity are present; the total transient growth based on the complete energy of the fluctuations must be used instead $\left(\right.$ cf. $\left.^{18,30}\right)$.

\section{PARAMETER STUDY}

In the following, two aspects contributing to non normality in the system will be investigated separately: the influence of the mean flow Mach number and flame-acoustic interaction via unsteady heat release. The configuration to be analyzed is simplified to a duct with a 1D flame, the isentropic nozzle being removed (see Fig. 8). The boundary conditions are set to $\hat{u}=0$ and $\hat{s}=0$ at the inlet and to $\hat{p}=0$ at the outlet of the domain. This means, that as soon as a mean flow is present, both acoustic energy and energy contained in entropy 


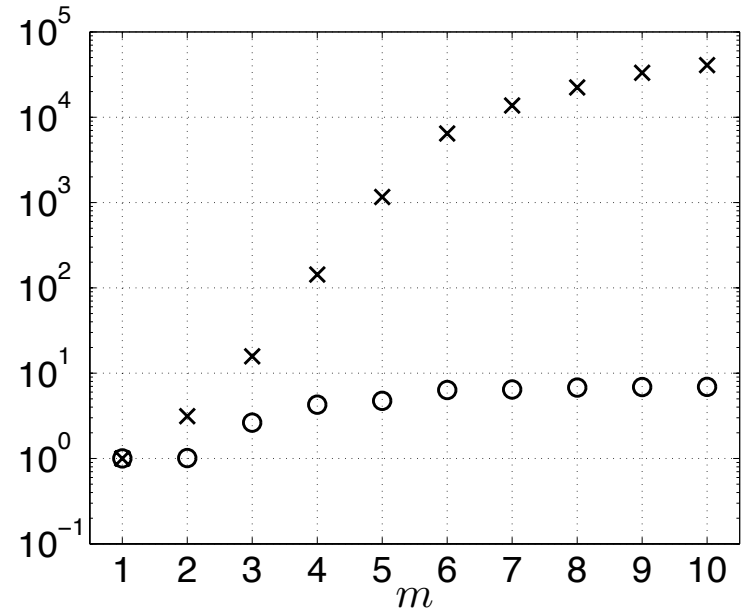

(a)Maximum possible amplification:

$$
\circ: G_{\max , t o t}^{m} ; \times: G_{\max , a c}^{m}
$$

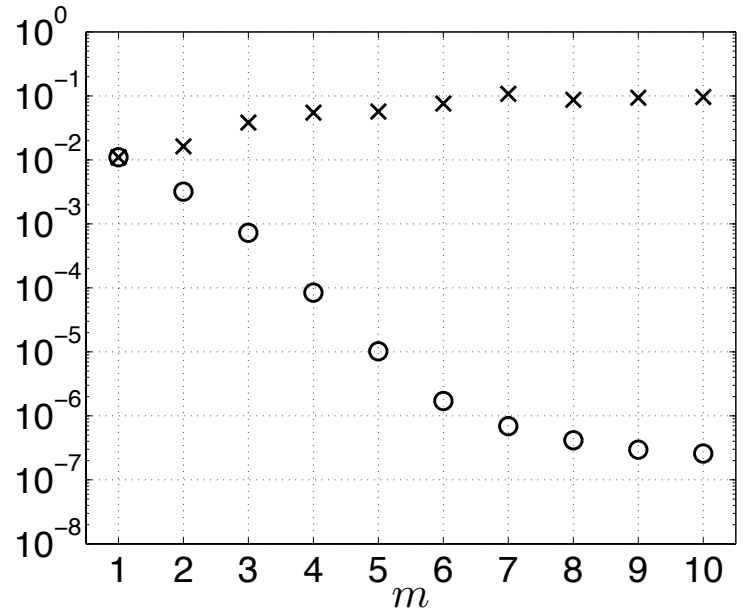

(b)Contribution of the acoustic energy to the optimal initial perturbation for $G_{\max , a c}^{m}$.

$$
\circ: \sigma(t=0) ; \times: \sigma\left(t=t_{\max }\right)
$$

FIG. 7. Dependence of transient growth on the number of eigenmodes $m$ retained for the analysis.

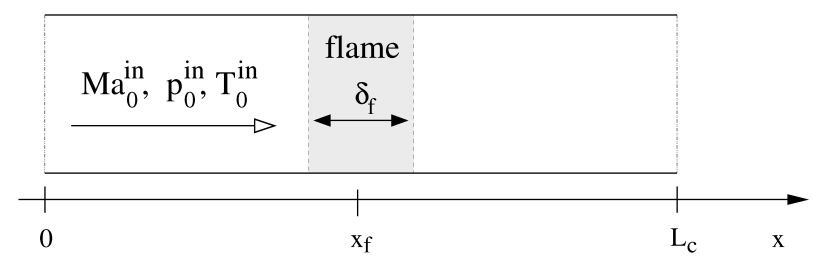

FIG. 8. Configuration used for Parameter Study.

fluctuations may leave the domain.

The flow parameters concerning temperature, pressure and gas constant of the fluid, as well as the position and thickness of the flame, remain those of Table I.

\section{A. Variation of mean flow Mach number}

In this subsection, the effect of mean flow Mach number on transient growth is explored. In particular, for inlet Mach numbers in the range from $M_{0}^{i n}=0.05$ to 0.20 , the maximum possible transient growth is computed based on the total disturbance energy, using up to eight eigenmodes of the configuration for modal expansion.

Cases with inlet Mach numbers smaller than $M_{0}^{i n}=0.05$ are not considered here, as for low 


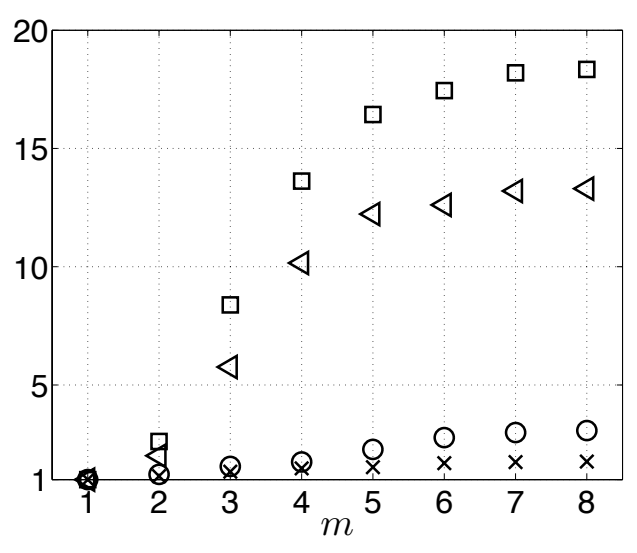

(a) $G_{\max }$ obtained by combining the first

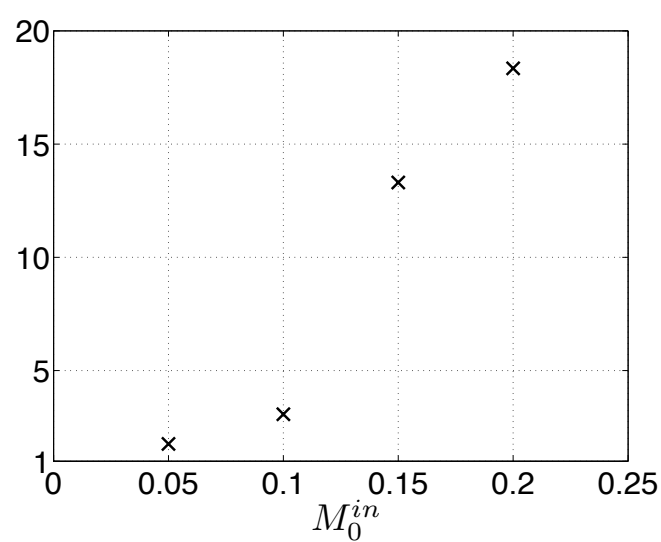

(b) $G_{\max }$ obtained for $m=8$

$m$ eigenvectors.

$\times: M_{0}^{i n}=0.05 ; \circ: M_{0}^{i n}=0.10 ;$

$\triangleleft: M_{0}^{i n}=0.15 ; \square: M_{0}^{i n}=0.20$

FIG. 9. Variation of $G_{\max }$ with the inlet Mach number.

mean flow Mach numbers the entropy fluctuations are structures with a wavelength much shorter than the acoustic wavelength, by a factor that corresponds to the mean flow Mach number $\lambda_{e} / \lambda_{a} c \approx u / c=M$. This implies that for low Mach numbers the computational grid has to be very fine in order to correctly capture the effect of entropy perturbations. At the same time, a demonstration of low Mach number computations is not the main concern here, as the effects of non normality are expected to decrease with the mean flow velocity.

The results for $G_{\max }^{m}$ as defined in Eq. (33) obtained by combining the first $m$ eigenmodes are plotted in Fig. 9(a). The plot confirms that the growth factor $G_{m a x}^{m}$ converges towards a stable value when about seven modes are included in the analysis. Figure 9(b) resumes the values of $G_{\max }$ obtained by combining eight eigenvectors as function of the inlet Mach number. As expected, non normal effects are relatively small at low Mach numbers, and increase with growing mean flow velocity. For $M_{0}^{i n}=0.05$, the maximum transient growth obtained is approximately $G_{\max } \approx 2.5$, which is considerably lower than the value obtained for the same velocity for the configuration including the choked nozzle. Yet, increasing the mean flow Mach number leads then to maximum possible growth factors as high as $G_{\max } \approx 18$ for $M_{0}^{i n}=0.20$. 


\section{B. Variation of the strength of the unsteady heat release term}

In the remainder of this section, the impact of unsteady heat release on non-normal interactions and resulting growth of perturbation energy is investigated. As before, the effects of non-normality are determined based on the total disturbance energy approach.

As introduced in section II, the unsteady heat release is described using an $n$ - $\tau$-model, leading to the expression stated in Eq. (6). In the frequency domain, this unsteady heat release term reads:

$$
\hat{q}(\mathbf{x})=\frac{q_{t o t a l}}{U_{b u l k}} H_{q}(\mathbf{x}) e^{i \omega \tau} \hat{\mathbf{u}}_{\mathbf{x}_{\text {ref }}} \cdot \mathbf{n}_{\text {ref }}
$$

The local interaction index $H_{q}$ can be linked to a parameter $n$ as follows:

$$
\begin{array}{lll}
H_{q}(x)=\frac{n}{\delta_{f}} \frac{U_{\text {bulk }}}{q_{\text {total }}} \frac{\gamma}{\gamma-1} p_{0} & & \text { for } \quad x_{f}-\frac{\delta_{f}}{2}<x<x_{f}+\frac{\delta_{f}}{2} ; \\
H_{q}(x)=0 & & \text { otherwise }
\end{array}
$$

where $x_{f}$ and $\delta_{f}$ denote the position and the thickness of the flame, respectively.

Varying the parameter $n$ hence allows to change the strength of the flame-acoustic interaction, which is what is investigated in the following. However, once unsteady heat release is introduced with $n>0$, some of the modes are expected to become linearly unstable. This is indeed observed for the configuration investigated here, see Table II, which shows a trend towards positive values for the imaginary part of all eigenfrequencies if the interaction index $n$ is increased. The analysis has been carried out at a relatively high inlet Mach number of $M_{i n}=0.2$, resulting in high losses of disturbance energy at the boundaries, and thus reducing both the growth rates and the number of unstable modes. Nevertheless, modes 4 and 5 are linearly unstable for $n>2$.

In such cases, the computation of a transient growth factor is not obviously meaningful, perhaps not even possible. However, the system being linearly unstable does not mean that non-orthogonal effects do not exist anymore, but rather that $G_{t o t}^{m}(t)$ cannot be used for the evaluation, as it will inevitably tend to infinity. For this reason confrontation with linearly unstable systems is usually avoided. Otherwise, an analysis can be made possible by introducing a damping term in the governing equations ${ }^{8}$. However, this approach requires changes to the formulation of the underlying eigenvalue problem, and is thus opposed to the idea of studying non-normal effects in a post-processing step.

In this paper a different strategy is therefore adopted: Simply speaking, the growth of perturbation energy including non-normal interactions is compared to the growth of the 


\begin{tabular}{|c|ccc|}
\hline $\mathrm{m}$ & $n=2$ & $n=3$ & $n=4$ \\
\hline 1 & $53.3-64.0 i$ & $45.3-58.3 i$ & $39.4-54.6 i$ \\
2 & $332.6-10.6 i$ & $342.5-5.4 i$ & $350.3-2.1 i$ \\
3 & $457.5-188.1 i$ & $440.9-169.6 i$ & $433.7-160.0 i$ \\
$\mathbf{4}$ & $662.6+41.5 i$ & $677.9+73.9 i$ & $696.7+103.6 i$ \\
$\mathbf{5}$ & $893.4+10.6 i$ & $877.5+26.6 i$ & $859.9+37.5 i$ \\
6 & $1114.1-204.7 i$ & $1069.6-194.2 i$ & $1045.4-186.2 i$ \\
7 & $1229.6-43.8 i$ & $1221.4-30.2 i$ & $1217.0-23.5 i$ \\
8 & $1567.4-37.7 i$ & $1404.3-143.3 i$ & $1415.2-124.1 i$ \\
9 & $1784.1-76.8 i$ & $1567.4-37.7 i$ & $1574.9-29.5 i$ \\
10 & $1933.8-33.5 i$ & $1805.0-68.8 i$ & $1819.0-60.9 i$ \\
\hline
\end{tabular}

TABLE II. Eigenfrequencies of first modes with flame-acoustic interaction $n>0$, time delay $\tau=0.5 \mathrm{~ms}$, inlet Mach number of $M_{i n}=0.2$. Mode index $m$ in bold face indicates linearly unstable modes.

energy of the most unstable eigenmode,

$$
E_{\text {max }}=E_{0} \exp \left(-2 \omega_{i}^{\max } t\right)
$$

Results are shown in Fig. 10. Is is evident that non-normal interactions can accelerate the growth, such that larger perturbation energies are reached at earlier times. However, such enhanced growth is only observed in the early stages of growth $t^{\prime}<0.2$, at later times the growth rate of the most unstable modes dominates the evolution. This becomes particularly obvious when the data shown in Fig. 10 is plotted on a log-linear scale (see Fig. 11). Then it becomes clear that for $n=2$ and $n=4$, perturbation energies including non-normal interactions are about four times and two times larger, respectively, than the energy that the most unstable mode reaches at the same time.

As mentioned before, for the case of a linearly unstable system $G(t)$ will tend to infinity and can therefore not be used for the analysis. In such conditions, the largest oscillation amplitudes are always observed at $t \rightarrow \infty$, and they would correspond to the most unstable mode, i.e. the mode with largest imaginary part $\omega_{i}$. 

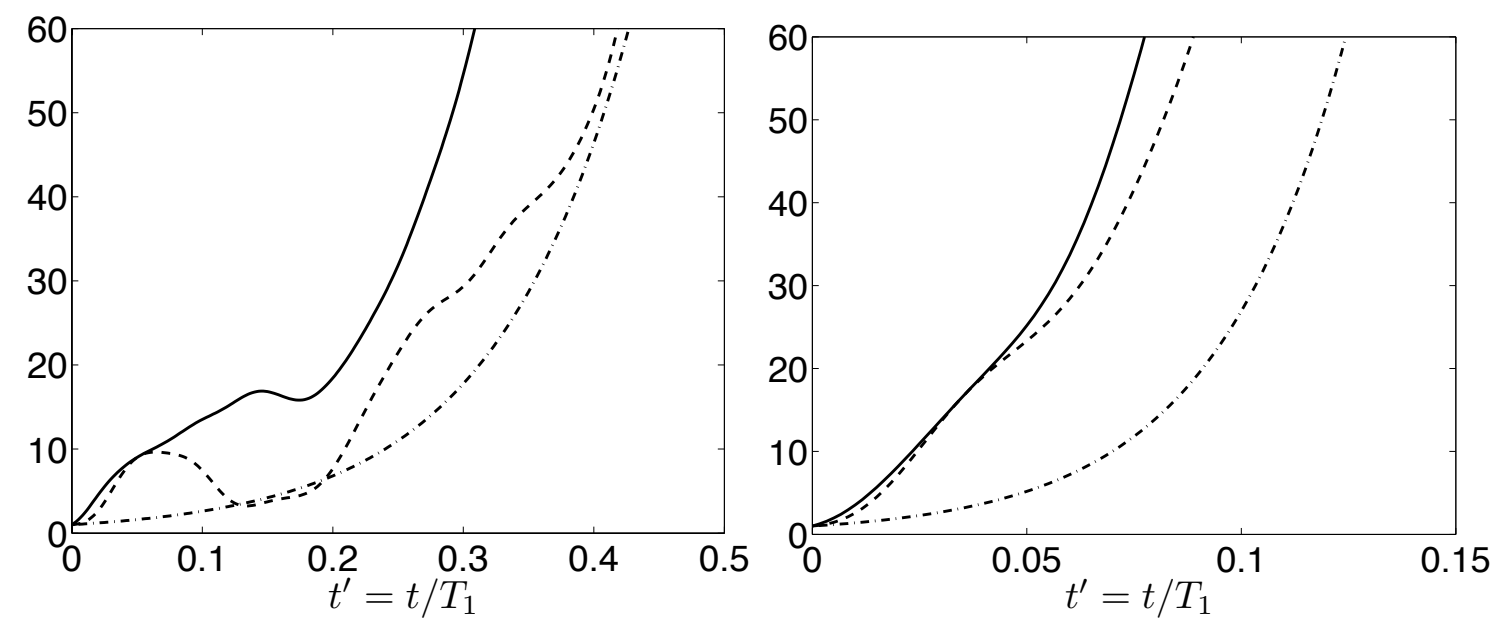

FIG. 10. Growth of perturbation energy with unsteady heat release $n=2$ (left) and $n=4$ (right) for $m=10$ eigenmodes. $-\cdot-\cdot-$ : most unstable mode $E_{\max }(t) ;-{ }_{-}: G(t)$ maximum growth; $---: E(t)$ growth from optimal initial condition
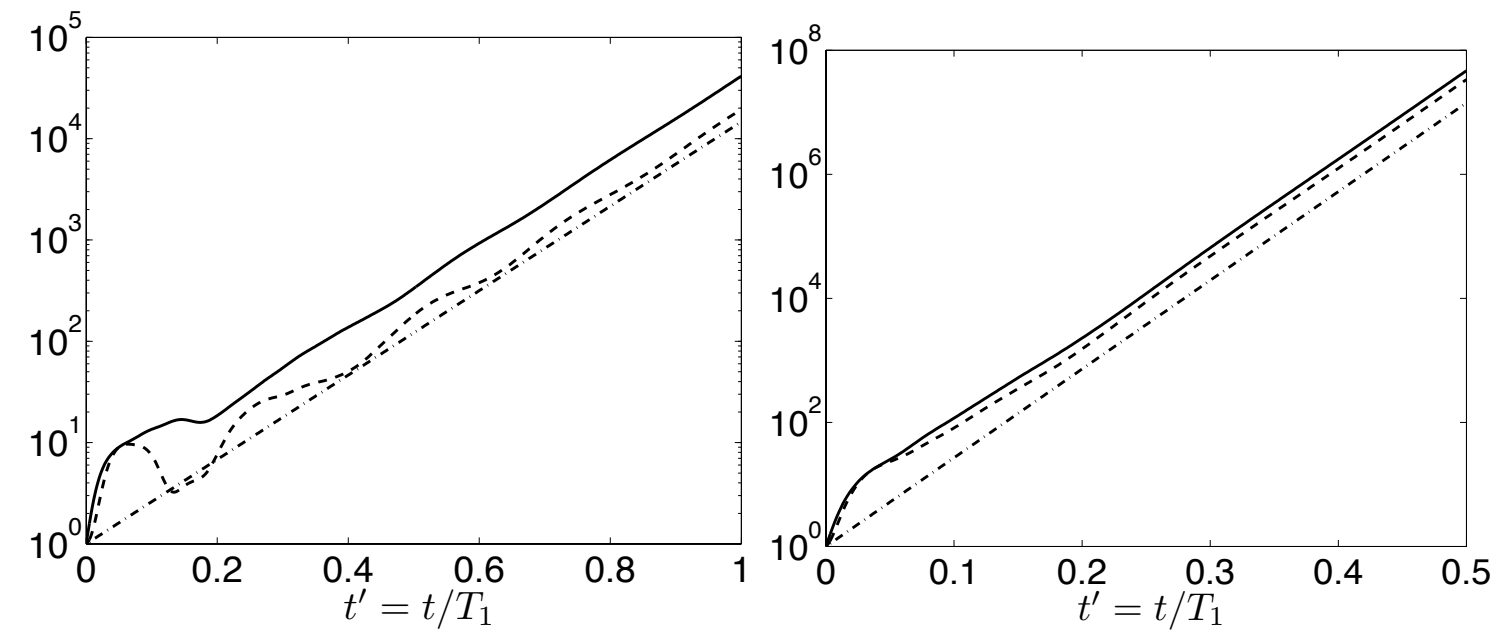

FIG. 11. Growth of perturbation energy with unsteady heat release $n=2$ (left) and $n=4$ (right), log-linear scale. $-\cdot-\cdot-$ : most unstable mode $E_{\max }(t) ;--: G(t)$ maximum growth; $---:_{E}(t)$ growth from optimal initial condition

In order to identify the optimal initial conditions and to quantify the effect of accelerated initial growth observed in Fig. 10, the approach used so far is slightly modified: The exponential growth of the term $G(t)$ is counteracted by multiplication with $\exp \left(-2 \omega_{i}^{\max } t\right)$, which leads to a modified growth term of the form

$$
G_{\max }^{c o m p} \equiv \max _{t}\left[G(t) \exp \left(-2 \omega_{i}^{\max } t\right)\right]
$$



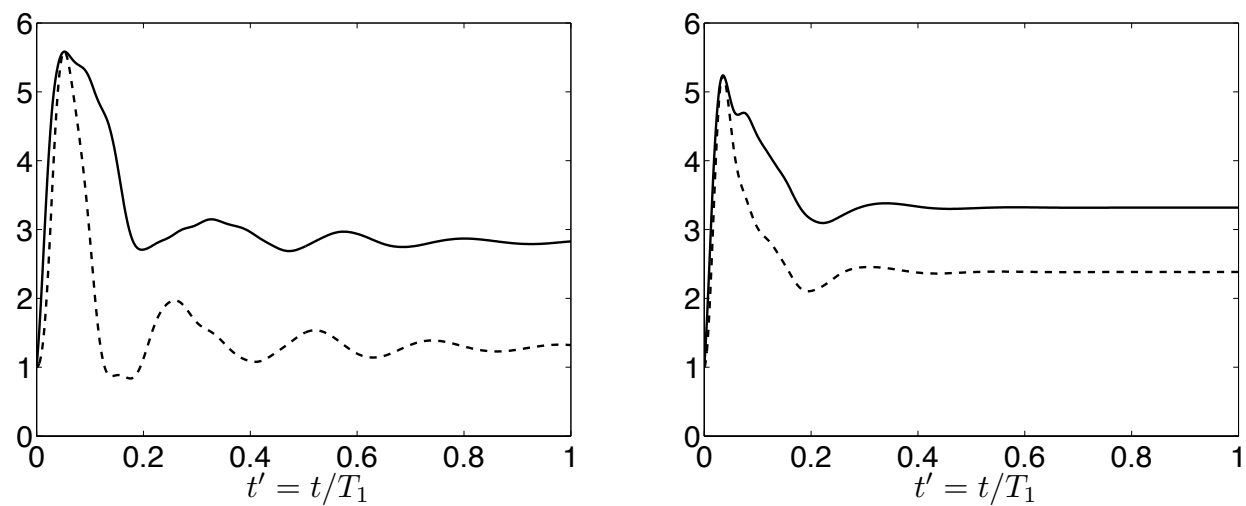

FIG. 12. Temporal evolution of the compensated terms for $n=2$ (left) and $n=4$ (right) for $m=10$ eigenmodes: $-\frac{-}{-} G(t) \exp \left(-2 \omega_{i}^{\max } t\right)---: E(t) \exp \left(-2 \omega_{i}^{\max } t\right)$

(where comp stands for compensation of the exponential growth.) In difference to $G(t)$, the growth term of Eq. 40 tends towards a stable value for large times, and presents a maximum value during the initial cycle. This "compensated" growth term can therefore be used to quantify the effect of accelerated growth in unstable non-orthogonal systems, the approach being otherwise the same as for linearly stable systems.

\section{CONCLUSION}

This article evaluates non-normal effects for a thermoacoustic system that contains both a source of entropy fluctuations and a zone of accelerated mean flow. Rather than using a singular value decomposition approach, the determination of the maximum transient energy growth and the corresponding optimal initial perturbation is carried out as a very efficient post-processing analysis based on an expansion in eigenmodes. These modes are obtained by solving the Linearized Euler Equations using a finite volume technique, a method which allows to take into account mean flow effects and is at the same time suitable for complex geometries. It should be noted, though, that as the approach is based on an eigenmode decomposition, the computation of eigenvectors being associated to discrete frequencies is a prerequisite to this analysis. The generalization of the presented technique from $1 \mathrm{D}$ to a complete 3D analysis based on the Linearized Euler Equations is limited to the case of discrete eigenmodes. For hydrodynamic instabilities associated to a continuous frequency spectrum, the presented approach may require further adjustments not considered in this 
study.

The results confirm that the eigenmodes of thermo-acoustic configurations are, in general, not orthogonal, which allows for transient growth of disturbance energy. However, it is pointed out that for the analysis of non-normal effects of such a configuration the definition of the norm, or equivalently the proper choice of the disturbance energy, is crucial. Two approaches are presented in this paper, the first one being based on an energy term that is equivalent to the classical acoustic energy; the second one being based on the total disturbance energy and therefore including the contribution of entropy fluctuations. It is shown that the use of the acoustic energy concept may cause misleading results in configurations that include mean flow effects: If the energy of entropy fluctuations is neglected in the analysis, any energy transfer from entropy to acoustic fluctuations will lead to spurious values for transient (acoustic) energy growth.

Applied to a schematic representation of a combustion chamber with a moderate mean flow speed and choked exit without unsteady heat release, the method presented predicts a maximum possible amplification of $G_{\max , t o t} \approx 6$. For this configuration, a linear combination of five to six eigenmodes is sufficient to determine the maximum possible amplification and the optimal initial perturbation. A subsequent parameter study shows the effects of the mean flow Mach number and unsteady heat release on the degree of non-normality. The analysis confirms that non normal effects increase with the mean flow velocity and the strength of the thermo-acoustic interaction. It is demonstrated also that linearly unstable systems can be analysed with the method presented. The results show that transient growth due to non-normality may lead to high amplitudes of perturbations at times earlier than those predicted by linear theory, i.e. before the exponential growth is the dominant aspect.

After completion of the work described in the present paper, Subramanian \& Sujith ${ }^{31}$ have proposed a norm for the energy of heat release fluctuations in a model of a laminar premixed flame. In support of the findings of the present study, it is found that the transient growth of energy detected in the system may be strongly influenced by the choice of norm. 


\section{ACKNOWLEDGMENTS}

K.W. and C.S. are grateful to the European Community for funding their PhD and PostDoc work under the projects AETHER (Contract No. FP6 - MRTN-CT-2006-035713) and MyPLANET (Contract No. FP7- PEOPLE - 2007-1-1-ITN -210781), respectively. This

work was also performed in the framework of the project MICCA funded by the Agence Nationale pour la Recherche (Contract No. ANR-08-BLAN-0027-01).

\section{Appendix A: Orthogonality of the Eigenmodes}

The objective of this appendix is to give a short derivation of Eq. (17), which is used to discuss the orthogonality of the eigenmodes.

Consider two eigenvectors $\hat{v}_{1}$ and $\hat{v}_{2}$, that are associated to the complex eigenfrequencies $\omega_{1}$ and $\omega_{2}$, respectively. A weighted inner product can then be defined as follows:

$$
\left\langle\hat{v}_{1} \mid \hat{v}_{2}\right\rangle_{W_{a c}}=\int_{V} \frac{1}{2 \gamma p_{0}} \hat{p}_{1} \hat{p}_{2}^{*} d V+\int_{V} \frac{\rho_{0}}{2} \hat{\mathbf{u}}_{1} \cdot \hat{\mathbf{u}}_{2}^{*} d V,
$$

where $V$ denotes the domain's volume, and $*$ stands for complex conjugates.

Writing the Helmholtz-Equation (cf. Eq. (7))for the complex conjugate of $\hat{p}_{2}$ yields:

$$
\frac{\hat{p}_{2}^{*}}{\gamma p_{0}}=-\frac{1}{\omega_{2}^{* 2}} \nabla \cdot \frac{1}{\rho_{0}} \nabla \hat{p}_{2}^{*}-i \frac{\gamma-1}{\gamma p_{0} \omega_{2}^{*}} \hat{q}_{2}^{*},
$$

The first integral on the RHS of Eq. A1 can thus be rewritten as:

$$
\int_{V} \frac{1}{2 \gamma p_{0}} \hat{p}_{1} \hat{p}_{2}^{*} d V=-\frac{1}{2 \omega_{2}^{* 2}} \int_{V} \hat{p}_{1} \nabla \cdot \frac{1}{\rho_{0}} \nabla \hat{p}_{2}^{*} d V-i \frac{1}{2 \omega_{2}^{*}} \int_{V} \frac{\gamma-1}{\gamma p_{0}} \hat{p}_{1} \hat{q}_{2}^{*} d V
$$

Using integration by parts, this result can be further developed into:

$$
\begin{aligned}
\int_{V} \frac{1}{2 \gamma p_{0}} \hat{p}_{1} \hat{p}_{2}^{*} d V & =\frac{1}{2\left(\omega_{1}^{2}-\omega_{2}^{* 2}\right)} \int_{S} \frac{1}{\rho_{0}}\left(\hat{p}_{1} \nabla \hat{p}_{2}^{*}-\hat{p}_{2}^{*} \nabla \hat{p}_{1}\right) \cdot \mathbf{n} d S \\
& +\frac{i}{2\left(\omega_{1}^{2}-\omega_{2}^{* 2}\right)} \int_{V} \frac{\gamma-1}{\gamma p_{0}}\left(\omega_{2}^{*} \hat{p}_{1} \hat{q}_{2}^{*}+\omega_{1} \hat{p}_{2}^{*} \hat{q}_{1}\right) d V
\end{aligned}
$$

where $S$ denotes the domain's boundary and $\mathbf{n}$ the unit vector normal to the boundary. The second integral on the LHS of Eq. (A1) can be rewritten using the relation $\rho_{0} \hat{\mathbf{u}}_{k}=$ $\frac{1}{i \omega_{k}} \nabla \hat{p}_{k}$, which yields:

$$
\int_{V} \frac{\rho_{0}}{2} \hat{\mathbf{u}}_{1} \cdot \hat{\mathbf{u}}_{2}^{*} d V=\frac{1}{2\left(\omega_{1} \omega_{2}^{*}\right)} \int_{V} \frac{1}{\rho_{0}} \nabla \hat{p}_{1} \cdot \nabla \hat{p}_{2}^{*} d V
$$


Further development using integration by parts leads to:

$$
\begin{aligned}
\frac{1}{2\left(\omega_{1} \omega_{2}^{*}\right)} \int_{V} \frac{1}{\rho_{0}} \nabla \hat{p}_{1} \cdot \nabla \hat{p}_{2}^{*} d V & =\frac{1}{2\left(\omega_{1}^{2}-\omega_{2}^{* 2}\right)} \int_{S} \frac{1}{\rho_{0}}\left(\frac{\omega_{1}}{\omega_{2}^{*}} \hat{p}_{1} \nabla \hat{p}_{2}^{*}-\frac{\omega_{2}^{*}}{\omega_{1}} \hat{p}_{2}^{*} \nabla \hat{p}_{1}\right) \cdot \mathbf{n} d S \\
& +\frac{i}{2\left(\omega_{1}^{2}-\omega_{2}^{* 2}\right)} \int_{V} \frac{\gamma-1}{\gamma p_{0}}\left(\omega_{1} \hat{p}_{1} \hat{q}_{2}^{*}+\omega_{2}^{*} \hat{p}_{2}^{*} \hat{q}_{1}\right) d V,
\end{aligned}
$$

Finally, adding the expressions given in Eqs. (A4) and (A6) yields the result discussed in section II:

$$
\left\langle\hat{v}_{1} \mid \hat{v}_{2}\right\rangle_{W_{a c}}=\frac{1}{2} \frac{1}{\omega_{1}-\omega_{2}^{*}}\left[\int_{S} \frac{1}{\rho_{0}}\left(\hat{p}_{1} \frac{\nabla \hat{p}_{2}^{*}}{\omega_{2}^{*}}-\hat{p}_{2}^{*} \frac{\nabla \hat{p}_{1}}{\omega_{1}}\right) \cdot \mathbf{n} d S+i \int_{V} \frac{\gamma-1}{\gamma p_{0}}\left(\hat{p}_{1} \hat{q}_{2}^{*}+\hat{p}_{2}^{*} \hat{q}_{1}\right) d V\right]
$$

\section{REFERENCES}

${ }^{1}$ F. Nicoud, L. Benoit, C. Sensiau, and T. Poinsot, "Acoustic modes in combustors with complex impedances and multidimensional active flames," AIAA Journal, 45, 426-441 (2007).

${ }^{2}$ K. Balasubramanian and R. I. Sujith, "Thermoacoustic instability in a Rijke tube: Nonnormality and nonlinearity," Phys. Fluids, 20, 044103-1-11 (2008).

${ }^{3}$ L. N. Trefethen, A. E. Trefethen, S. C. Reddy, and T. A. Driscoll, "Hydrodynamic stability without eigenvalues," Science, 261, 578-584 (1993).

${ }^{4}$ D. Henningson and P. Schmid, "Vector eigenfunction expansions for plane channel flows," Stud. Appl. Math., 87 (1992).

${ }^{5}$ P. J. Schmid and D. S. Henningson, Stability and Transition in Shear Flows (Springer, New York, 2001).

${ }^{6}$ J. Lim and J. Kim, "A singular value analysis of boundary layer control," Phys. Fluids, 16 (2004).

${ }^{7}$ F. E. C. Culick, "Combustion instabilities in liquid-fueled propulsion systems - An overview," in AGARD Conference Proceedings No. 450, 72B Specialists' Meeting of the AGARD Propulsion and Energetics Panel (1988).

${ }^{8}$ S. Nagaraja, K. Kedia, and R. I. Sujith, "Characterizing energy growth during combustion instabilities: Singularvalues or eigenvalues?" Proc. Comb. Inst., 32, 2933-2940 (2009).

${ }^{9}$ F. Nicoud and K. Wieczorek, "About the zero mach number assumption in the calculation of thermoacoustic instability," International Journal of Spray and Combustion Dynamic, 1, 67-112 (2009). 
${ }^{10}$ K. Balasubramanian and R. I. Sujith, "Non-normality and nonlinearity in combustion acoustic interaction in diffusion flames," J. Fluid Mech., 594, 29-57 (2008).

${ }^{11}$ F. Selimefendigil, R. Sujith, and W. Polifke, "Identification of heat transfer dynamics for non-modal analysis of thermoacoustic stability," Applied Mathematics and Computation, 217, 5134-5150 (2011), ISSN 0096-3003.

${ }^{12}$ H. Landau, "On Szegö's eigenvalue distribution theorem and non-Hermitian kernels," Journal d'Analyse Mathématique, 28, 335-357 (1975).

${ }^{13}$ L. N. Trefethen, "Computation of pseudospectra," Acta Numerica, 247-295 (1999).

${ }^{14}$ C. Sensiau, F. Nicoud, and T. Poinsot, "A tool to study azimuthal and spinning modes in annular combustors," Int. Journal Aeroacoustics, 8, 57-68 (2009).

${ }^{15}$ E. Gullaud, S. Mendez, C. Sensiau, F. Nicoud, and T. Poinsot, "Effect of multiperforated plates on the acoustic modes in combustors," C. R. Acad. Sci.Mécanique, 337, 406-414 (2009).

${ }^{16}$ A. P. Dowling, "The calculation of thermoacoustic oscillations," J. Sound Vib., 180, 557-581 (1995).

${ }^{17}$ T. Gebhardt and S. Grossmann, "Chaos transition despite linear stability," Physical Review E, 50, 3705 (1994).

${ }^{18} \mathrm{~S}$. Mariappan and R. Sujith, "Thermoacoustic instability in a solid rocket motor - nonnormality and nonlinear instabilities," J. Fluid Mech., 653, 1-33 (2010).

${ }^{19}$ T. Lieuwen and V. Yang, "Combustion Instabilities in Gas Turbine Engines. Operational Experience, Fundamental Mechanisms and Modeling," in Prog. in Astronautics and AeronauticsAIAA, Vol. 210 (2005).

${ }^{20}$ T. Poinsot and D. Veynante, Theoretical and Numerical Combustion (R.T. Edwards, 2nd edition, 2005).

${ }^{21}$ L. Crocco, "Aspects of combustion instability in liquid propellant rocket motors. Part I," J. American Rocket Society, 21, 163-178 (1951).

${ }^{22}$ L. Crocco, "Aspects of combustion instability in liquid propellant rocket motors. Part II," J. American Rocket Society, 22, 7-16 (1952).

${ }^{23}$ N. Noiray, D. Durox, T. Schuller, and S. Candel, "A unified framework for nonlinear combustion instability analysis based on the flame describing function." J. Fluid Mech., 615, 139-167 (2008).

${ }^{24}$ M. K. Myers, "Transport of energy by disturbances in arbitrary steady flows," J. Fluid 
Mech., 226, 383-400 (1991).

${ }^{25}$ N. Karimi, M. Brear, and W. Moase, "Acoustic and disturbance energy analysis of a flow with heat communication," J. Fluid Mech., 597, 67-89 (2008).

${ }^{26}$ A. Giauque, T. Poinsot, M. Brear, and F. Nicoud, "Budget of disturbance energy in gaseous reacting flows," in Proc. of the Summer Program (Center for Turbulence Research, NASA Ames/Stanford Univ., 2006) pp. 285-297.

${ }^{27}$ W. Polifke, C. O. Paschereit, and K. Döbbeling, "Constructive and destructive interference of acoustic and entropy waves in a premixed combustor with a choked exit," Int. J. of Acoustics and Vibration, 6, 135-146 (2001).

${ }^{28}$ F. E. Marble and S. Candel, "Acoustic disturbances from gas nonuniformities convected through a nozzle," J. Sound Vib., 55, 225-243 (1977).

${ }^{29}$ F. Nicoud and T. Poinsot, "Thermoacoustic instabilities: should the Rayleigh criterion be extended to include entropy changes ?" Combust. Flame, 142, 153-159 (2005).

${ }^{30}$ S. Mariappan, P. Schmid, and R. Sujith, "Role of transient growth in subcritical transition to thermoacoustic instablitiy in a horizontal Rijke tube," in 16th AIAA/CEAS Aeroacoustics Conference, Stockholm, Sweden, June 7-9, 2010, AIAA 2010-3857 (2010).

${ }^{31}$ P. Subramanian and R. Sujith, "Non-normality and internal flame dynamics in premixed flame-acoustic interaction," J. Fluid Mech., 679, 315-342 (2011). 